\title{
NEUTRON DOSE RATE AT THE SwissFEL INJECTOR TEST FACILITY: FIRST MEASUREMENTS
}

\author{
E. Hohmann*, N. Frey, A. Fuchs, C. Harm, H. Hödlmoser, R. Lüscher, S. Mayer, O. Morath, R. Philipp, \\ A. Rehmann and T. Schietinger \\ Paul Scherrer Institute, 5232 Villigen - PSI, Switzerland \\ *Corresponding author: eike.hohmann@psi.ch
}

\begin{abstract}
At the Paul Scherrer Institute, the new SwissFEL Free Electron Laser facility is currently in the design phase. It is foreseen to accelerate electrons up to a maximum energy of $7 \mathrm{GeV}$ with a pulsed time structure. An injector test facility is operated at a maximum energy of $300 \mathrm{MeV}$ and serves as the principal test and demonstration plant for the SwissFEL project. Secondary radiation is created in unavoidable interactions of the primary beam with beamline components. The resulting ambient doseequivalent rate due to neutrons was measured along the beamline with different commercially available survey instruments. The present study compares the readings of these neutron detectors (one of them is specifically designed for measurements in pulsed fields). The experiments were carried out in both, a normal and a diagnostic mode of operation of the injector.
\end{abstract}

\section{INTRODUCTION}

The Paul Scherrer Institute is a Swiss national research laboratory with a focus on large-scale research facilities. As part of the future SwissFEL Free Electron Laser facility $^{(1)}$, an injector test facility has been in operation since 2010. It serves as principal test bed and demonstration plant to determine and confirm the basic requirements of the accelerator regarding the quality of the electron beam, as well as to optimise components and procedures. Secondary radiation is created in unavoidable interactions of the primary beam with beamline components. During normal operation, the beam is optimised for small emittance to achieve the FEL-pulse requirements. This results in small losses, negligible from a radiation protection point of view. However, electrons produced spontaneously due to field emission in the photocathode or in the accelerating cavities (referred to as dark current) are also to be considered. Since the accelerator is optimised for the FEL pulses, the dark current not originating from the gun is expected to be lost close to the nearest downstream magnet and therewith limited in energy. The dark current from the gun can be propagated through the machine and is expected to be lost mainly in a magnetic chicane used for bunch compression. In the beam optimisation process, diagnostic screens may be inserted into the beam path, also leading to secondary radiation.

In this study, the neutron ambient dose-equivalent rate (subsequently referred to as dose rate) arising during normal and diagnostic mode of operation was measured with different commercially available survey instruments.

\section{EXPERIMENTAL SET-UP}

From a radiation protection point of view, the accelerator can be divided into five sections. In first section, electrons are created by an electron gun due to laserinduced photo emission. These electrons will be accelerated in four successive RF cavities (operated at S-band frequencies) to an energy of $230 \mathrm{MeV}$ used for this study. A magnetic chicane with a nominal angle of $4^{\circ}$ is used to reduce the length of the photo electron pulse. This bunch compressor is followed by a diagnostic section with several devices and magnets. The beam is finally dumped in a steel block shielded by concrete (beam dump region).

Since the beam is optimised for transmission, the bunch length of the photo-induced electrons is $\sim 6 \mathrm{ps}$ (FWHM). The photo-electron current was measured with 14 beam monitors distributed along the beamline. Since their readings deviate $<2 \%$, a mean value from all monitors was used as a reference. The accelerator was operated at a repetition rate of $10 \mathrm{~Hz}$.

Dose rate measurements were carried at beam height at a distance of $1 \mathrm{~m}$, for different positions along the beamline (exemplarily showed in Figure 1) for the two different modes of operation.

\section{Normal mode of operation}

During normal operation, the beam was optimised for small emittance to achieve the FEL-pulse requirements. This results in losses of $<1 \%$, negligible from a radiation protection point of view. The mean charge of the photo electrons per pulse was $200 \mathrm{pC}$. The RF cavities are active for $\sim 1 \mu$ s during each cycle and therewith transport also the dark current through the accelerator, leading to additional secondary radiation.

\section{Diagnostic mode of operation}

For machine tuning and optimisation, luminescent screens made of LuAlO (thickness of $200 \mu \mathrm{m}$ ) or Si 


\section{E. HOHMANN ET AL.}

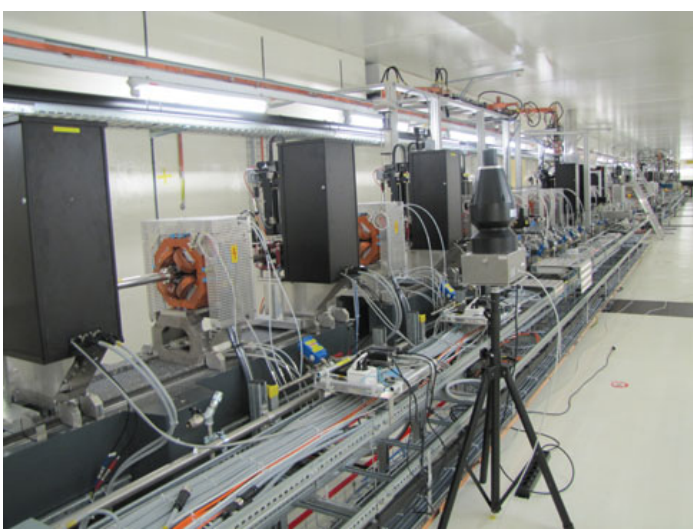

Figure 1. Experimental set-up exemplarily described by the diagnostic section of the accelerator with measurement position 'Diag' and the LB6419 neutron detector.

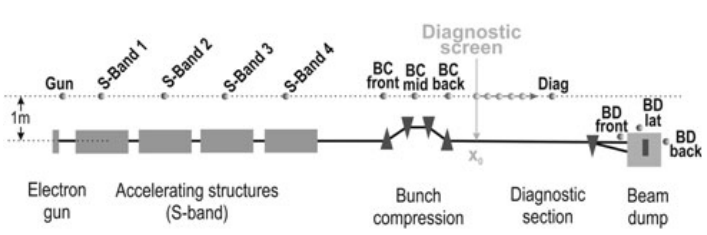

Figure 2. Schematic accelerator layout with measurement positions (bold face) for normal (black) and diagnostic mode of operation (grey).

(thickness of $300 \mu \mathrm{m}$ ), referred to as type 1 and 2, respectively, can be inserted in the beamline. This increases the emittance of the primary beam and leads to interaction with beamline components further downstream. For the present study, a screen positioned in the diagnostic section behind the bunch compressor was used. The expected beam loss should therewith arise mainly from the FEL pulses. The dose was measured from the position of the screen $\left(x_{0}\right)$ onwards and up to $14 \mathrm{~m}$ downstream (grey in Figure 2). The mean charge of the photo electrons per pulse was reduced to $135 \mathrm{pC}$.

A description of the accelerator and the measurement positions is given in Figure 2.

\section{NEUTRON DETECTORS}

For this study, three different types of commercially available survey instruments were used.

\section{LB6411}

The neutron probe LB6411 from Berthold Technologies is a rem counter using a spherical PE moderator with a borate shell and a ${ }^{3} \mathrm{He}$-proportional counter. It is optimised for an energy range from thermal to $20 \mathrm{MeV}^{(2)}$. This instrument is routinely used at PSI. The dose rate was recorded at a rate of $1 \mathrm{~Hz}$.

\section{WENDI-II}

The Thermo Wide Energy Neutron Detection Instrument (WENDI-II) is an extended-range rem counter designed for an energy range from thermal to $5 \mathrm{GeV}^{(3)}$. It consists of a ${ }^{3} \mathrm{He}$-proportional counter surrounded by a cylindrical polyethylene moderator assembly and a layer of tungsten powder. This additional layer of high- $\mathrm{Z}$ material enhances the detector response to neutrons with energies of $>8 \mathrm{MeV}$, due to the generation of secondary neutrons via $(n, x n)$ inelastic scattering reactions. During the measurement campaign, the dose rate was recorded at a rate of $1 \mathrm{~Hz}$ by a Thermo FH $40 \mathrm{G}$ survey meter.

\section{LB6419}

The LB6419 from Berthold Technologies is a compact survey instrument with a design suited for use in either continuous or pulsed fields. It employs two different detectors: a ${ }^{3} \mathrm{He}$-proportional counter with $\mathrm{PE}$ moderator (suitable for measurements in continuous fields with energies up to $20 \mathrm{MeV}$ ) and a plastic scintillator ${ }^{(4)}$. For this study, two channels were used: the ${ }^{3} \mathrm{He}$-proportional counter (referred to as LB6419cont) and the scintillator reading from the ${ }^{12} \mathrm{C}(\mathrm{n}, \mathrm{p}){ }^{12} \mathrm{~B}$-reaction (referred to as LB6419pulsed). With this latter channel, only neutrons with energies of $>20 \mathrm{MeV}$ and a pulsed time structure can be detected.

All detectors were calibrated in terms of ambient dose-equivalent $H^{*}(10)$ rate using a ${ }^{252} \mathrm{Cf}$-neutron source, with the exception of the LB6419pulsed, which was calibrated at the CERF facility ${ }^{(5)}$.

\section{DATA ANALYSIS}

For each position, all detectors were irradiated for at least $500 \mathrm{~s}$. During this period, the mean charge $Q$ recorded by the beam monitors every $0.1 \mathrm{~s}$ varies $<3 \%$. From these values, a mean dose $D_{\text {pulse }}$ per pulse with statistical uncertainty was evaluated (Figure 3) and normalised to a mean charge per pulse:

$$
D_{\text {Pulse }}=\frac{<D(t)>_{\Delta t}}{<Q(t)>_{\Delta t}} .
$$

The main uncertainty of the measured doses in the instruments dedicated energy range arises from their energy-dependent dose response, which is estimated with $30 \%$, according to the manufacturers' specifications. The contribution of both uncertainties (from the mean dose value and the mean photo-electron charge 


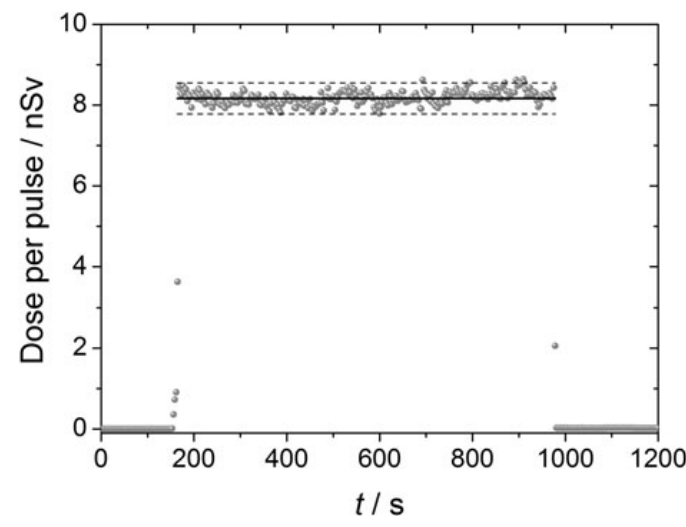

Figure 3. The mean value (solid line) with statistical uncertainty (dashed line) was evaluated for each measurement exemplarily showed for the WENDI-II reading recorded in intervals of 1 to $10 \mathrm{~s}$ (grey).

per pulse) is added in quadrature. Since the counting statistics of the LB6419pulsed depend strongly on the pulse identification and the time structure, the measurement uncertainty was estimated only arising from its energy response ${ }^{(6)}$.

\section{RESULTS}

To compare the readings (and therewith the impact of the pulsed radiation during routine operation), the registered values were normalised to a photo-electron charge of $200 \mathrm{pC}$ per pulse, the nominal value in the normal operation mode.

\section{Normal mode of operation}

During normal mode of operation, the dose per pulse increases from the gun towards the bunch compressor to a maximum value of $\sim 8 \mathrm{nSv}$ per pulse. Beyond that point, the dose per pulse decreases. The highest doses of $\sim 16 \mathrm{nSv}$ per pulse were measured laterally of the beam dump. Due to the substantial shielding (standardised concrete blocks) at the back of the beam dump, only low dose rates were measured behind it.

The WENDI-II is also sensitive to neutrons with energies of $>20 \mathrm{MeV}$. Its measurements indicate the highest measured dose. The LB6419pulsed is only sensitive to neutrons with energies of $>20 \mathrm{MeV}$ and depends strongly on a sufficient dose to trigger ${ }^{(6)}$; it may therefore underestimate the dose or even not register any. Since the internal data evaluation of this channel is not described in detail in the literature, only the uncertainty due to the energy dependence is taken into account. In the beam dump region, where the dose mainly arises from the FEL pulses (bunch length: $6 \mathrm{ps}$ ), it shows a significant increase.

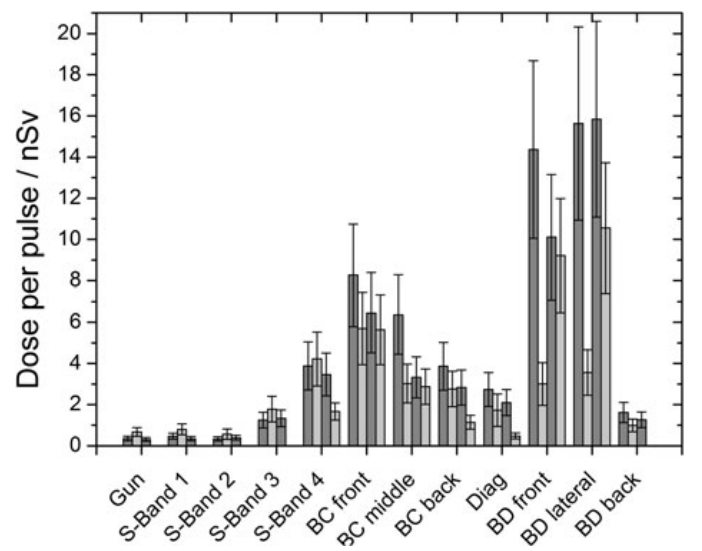

Figure 4. Measured doses normalised to a photo-electron charge of $200 \mathrm{pC}$ per pulse, with the WENDI-II (first bar from left), LB6411 (second bar), LB6419cont (third bar) and LB6419pulsed (fourth bar), respectively. Since the blackLB6419pulsed is only sensitive to neutrons with energies of $>20 \mathrm{MeV}$, some measurement positions show no reading on this detector.

Taking the various properties of the different detectors into account, their readings show good agreement. The dose values measured during normal mode of operation are summarised in Figure 4.

\section{Diagnostic mode of operation}

For radiation protection purposes, it is desirable to determine the dose due to neutrons with energies of $>20 \mathrm{MeV}$ (e.g. to define adequate local shielding, suitable for losses expected during a diagnostic mode of operation). Specific measurements were carried out with the LB6419cont and the WENDI-II. Both devices show comparable saturation effects during measurements in pulsed neutron fields ${ }^{(7)}$ but have a different sensitivity to high-energy neutrons (as described in the section Neutron detectors). The neutron dose arising from normal operation was subsequently subtracted from the registered values. For this study, the photo-electron current was reduced by $35 \%$ to decrease the impact of saturation effects of the measurement devices.

The neutron dose arising from inserting a screen of type 1 or 2 in the beam path is distributed over a distance of $\sim 14 \mathrm{~m}$ with a maximum dose of $35 \pm 9$ or $26 \pm 6 \mathrm{nSv}$ per pulse, respectively. The distance of the maximum measured dose per pulse according to the screen position depends also on screen type (Figure 5). This effect is caused by the different material and thickness of each screen type as described in the section Diagnostic mode of operation. A ratio of $1.4 \pm 0.2$ was observed comparing the dose registered with the detector suitable for high-energy neutrons 


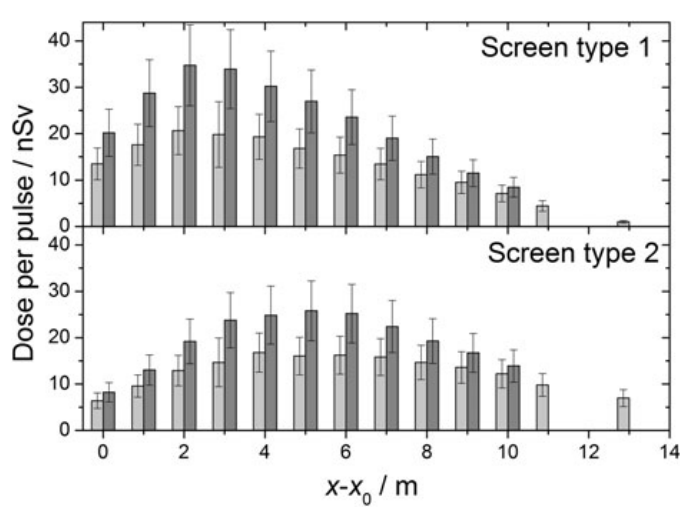

Figure 5. With the WENDI-II (first bar from left), LB6419cont (second bar) measured doses arising from inserting a screen type 1 (top) and type 2 (bottom) into the beam path. The results are normalised to a charge of $200 \mathrm{pC}$ per pulse.

(WENDI-II) or the LB6419cont black (suitable only for neutrons with energies of $<20 \mathrm{MeV}$ ).

\section{CONCLUSIONS}

Measurements were taken at the SwissFEL injector test facility using three different types of commercially available survey instruments for normal and diagnostic mode of operation at different positions inside the accelerator vault.

During normal operation, the doses indicated by the different instruments agree within the measurement uncertainty except for the beam dump region. There, due to its limited energy range and high sensitivity, the LB6411 shows significantly lower dose values than the other instruments. The photon background in the vault associated with each pulse causes the scintillator used by the LB6419 to saturate. As a result, only the channel using the delayed
${ }^{12} \mathrm{C}(\mathrm{n}, \mathrm{p}){ }^{12}$-reaction could be used during the measurements. The highest doses per pulse were measured next to the beam dump and the bunch compressor.

For the optimisation of the accelerator, luminescent screens can be inserted into the beam path causing a dose distributed over several metres depending on the screen type. The dose arise to $40 \%$ from neutrons with energies of $>20 \mathrm{MeV}$. Although the charge of each pulse were reduced to decrease effects due to saturation, the measured values call for further investigation.

\section{ACKNOWLEDGEMENTS}

The authors thank the PSI accelerator staff, in particular Bennie Smit, for the support and help.

\section{REFERENCES}

1. Ganter, R. Ed. SwissFEL conceptual design report, PSI10-04. Available on http://www.psi.ch/swissfel/ (2012).

2. Burgkhardt, B., Fieg, G., Klett, A., Plewnia, A. and Siebert, B. R. L. The neutron fluence and $\mathrm{h} *(10)$ response of the new LB6411 REM counter. Radiat. Prot. Dosim. 70, 361-364 (1997).

3. Olsher, R. H., Hsu, H.-H., Beverding, A., Kleck, J. H., Casson, W. H., Vasilik, D. G. and Devine, R. T. WENDI: an improved neutron rem meter. Health Phys., 79, 170-181 (2000).

4. Klett, A., Leuschner, A. and Tesch, N. A dose meter for pulsed neutron fields. Radiat. Meas. 45, 1242-1244 (2010).

5. Mitarof, A. and Silari, M. The cerneu high-energy reference field (cerf) facility for dosimetry at commercial flight altitudes and in space. Radiat. Prot. Dosim. 102, 7-22 (2002).

6. Leuschner, A. Private communication: Basic principles of the LB6419, DESY, Hamburg (2013).

7. Caresana, M. et al. Intercomparison of radiation protection instrumentation in a pulsed neutron field. Nucl. Inst. Methods in Phys. Res. A 737, 203-213 (2014). 